\title{
Asymptotic behavior of attracting and quasi-invariant sets of impulsive stochastic partial integrodifferential equations with delays and Poisson jumps
}

\author{
K. Ramkumar ${ }^{a, *}$, K. Ravikumar ${ }^{\mathrm{a}}$, Dimplekumar Chalishajar ${ }^{\mathrm{b}}$, A. Anguraj ${ }^{\mathrm{a}}$ \\ ${ }^{a}$ Department of Mathematics, PSG College of Arts and Science, Coimbatore, 641 046, India. \\ ${ }^{b}$ Department of Mathematics and Computer science, Mallory Hall, Virginia Military Institute, Lexington, VA 24450, USA.
}

\begin{abstract}
This paper is concerned with a class of impulsive stochastic partial integrodifferential equations (ISPIEs) with delays and Poisson jumps. First, using the resolvent operator technique and contraction mapping principle, we can directly prove the existence and uniqueness of the mild solution for the system mentioned above. Then we develop a new impulsive integral inequality to obtain the global, both $\mathrm{p}^{\text {th }}$ moment exponential stability and almost surely exponential stability of the mild solution is established with sufficient conditions. Also, a numerical example is provided to validate the theoretical result.
\end{abstract}

Keywords: Exponential stability, almost surely exponential stability, mild solution, attracting set, quasi-invariant set, Poisson jumps, resolvent operator.

2020 MSC: 60H10, 93E03, 35B35, 39B82.

(C)2021 All rights reserved.

\section{Introduction}

Functional differential equations appear as mathematical models in medicine, electrical engineering, biology, ecology, etc. The deterministic models regularly fluctuate because of noise which is random or at least appears to be so. Therefore, researchers moved from deterministic problems to stochastic ones. Differential and integral equations involving random variables play an important role in describing many physical, biological, engineering problems. Stochastic differential equations (SDEs) are important from the viewpoint of applications since they incorporate randomness into mathematical description of phenomena, thereby describing it more accurately. In recent years, a lot of interesting properties of the solution for the system such as existence, uniqueness, and stability have been obtained (see, [3-5, 7-9, 19] and the reference therein).

Besides delay effects, impulsive effects likewise exist in a wide variety of evolutionary processes, in which states are changed abruptly at certain moments of time, involving such fields as medicine, biology, economics, electronics and telecommunications, etc. Many interesting results on impulsive effects have

\footnotetext{
*Corresponding author

Email addresses: ramkumarkpsg@gmail.com (K. Ramkumar), ravikumarkpsg@gmail .com (K. Ravikumar), chalishajardn@vmi.edu (Dimplekumar Chalishajar), angurajpsg@yahoo.com (A. Anguraj)

doi: $10.22436 /$ jnsa.014.05.04
}

Received: 2020-12-08 Revised: 2020-12-30 Accepted: 2021-01-15 
been obtained [1, 2, 7, 15]. Furthermore, under impulsive perturbation, an equilibrium point sometimes does not exist in many physical systems, especially, in non-linear and nonautonomous dynamical systems and all solutions may gradually approach a bounded set called the attracting set. Therefore, an interesting subject is to discuss the two sets (1) Global attracting set and (2) Quasi-invariant set of dynamical systems with the impulsive effects. For more details, we refer to (see, $[12,16,18,23]$ and the references therein). In the past decades, a lot of interesting manuscripts are studied on global attracting and invariant set of stochastic dynamical systems. Among others, motivated by Chan's work [7], Long et al. [20] examined the global and exponential stability of impulsive stochastic neutral evolution system. Li [17] discussed the global attracting set and quasi-invariant set of impulsive neutral stochastic functional partial differential equations driven by fractional Brownian motion ( $\mathrm{fBm}$ ). In very recent years, a few works have been done on the qualitative properties of solutions for attracting set and quasi-invariant set of SDEs system, readers are referred to $[6,10,13,22]$.

In financial modeling, SDEs with jumps are often used to describe the dynamics of state variables such as credit ratings, stock indices, interest rates, exchange rates, and electricity prices [3, 9]. The jump component can capture event-driven uncertainties, such as corporate defaults, operational failures, (or) central bank announcements. On the other hand, the Poisson jumps have become a very popular in recent years, the Poisson jumps are generally based on the Poisson random measure in aspects of applications in many real life phenomena such as biology, finance and other field of science, see [14, 21]. Hence the analysis of the qualitative behavior of SDEs with Poisson jumps has attracted many researchers in recent years, see $[1,12,13]$. That is to say, there is no study on global attracting set and exponential stability of impulsive SDEs with Poisson jumps in the existing literature. All these facts push us to develop and explore techniques and methods for the global attracting set, both $\mathrm{p}^{\text {th }}$ moment exponential stability and almost surely exponential stability of SDEs and Poisson jumps, especially with delayed impulses.

Based on the above statement and analysis, this work is devoted to deriving the global attracting set, both $p^{\text {th }}$ moment exponential stability and almost surely exponential stability, of ISPIEs with Poisson jumps. We use the resolvent operator technique, impulsive integral inequality and stochastic analysis theory to attain this goal. The main improvement and superiority of this work can be highlighted as below.

(i) The global attracting set, both $\mathrm{p}^{\text {th }}$ moment exponential stability and almost surely exponential stability of ISPIEs with Poisson jumps are obtained.

(ii) We establish a new impulsive integral inequality, and this key inequality is applicable to investigate the global attracting set, both $p^{\text {th }}$ moment exponential stability and almost surely exponential stability of ISPIEs with Poisson jumps.

The rest of this paper is organized as follows. Section 2 deals with the notations and preliminaries, existence of mild solution is proved in Section 3. The global attracting set, both $\mathrm{p}^{\text {th }}$ moment exponential stability and almost surely exponential stability of ISPIEs with Poisson jumps are obtained in Section 4. Finally, an example is provided to illustrate the results in Section 5.

\section{Model description}

In this section, we have focus on the following ISPIEs with Poisson jumps:

$$
\begin{aligned}
d x(t)= & {\left[\mathfrak{A} x(t)+\int_{0}^{t} \mathfrak{B}(t-s) x(s) d s+\mathfrak{f}(t, x(t-\rho(t)))\right] d t+\mathfrak{g}(t, x(t-\delta(t))) d w(t) } \\
& +\int_{\mathfrak{Z}} \mathfrak{h}(t, x(t-\sigma(t)), \eta) \widetilde{\mathfrak{N}}(d t, d \eta), t \geqslant 0, t \neq t_{k}, \\
\Delta x\left(t_{k}\right)= & I_{k}\left(x_{k}^{-}\right), t=t_{k}, k=1,2, \ldots, \\
x_{0}(s)= & \varphi \in \mathscr{P} \mathscr{C}_{\mathfrak{F}_{0}}^{b}([-r, 0], \mathbb{H}), s \in[-r, 0],
\end{aligned}
$$


where $\mathfrak{A}: \mathscr{D}(\mathfrak{A}) \subset \mathbb{H} \rightarrow \mathbb{H}$ is the infinitesimal generator of a $\mathrm{C}_{0}$-semigroup $\{\mathrm{S}(\mathrm{t})\}_{\mathfrak{t} \geqslant 0}$ on Hilbert space $\mathbb{H}$ with inner product $\langle\cdot, \cdot>$ and norm $\|\cdot\|, \mathfrak{B}(\mathrm{t})$ a close linear operator on $\mathbb{H}$ with $\mathscr{D}(\mathfrak{A}) \subset \mathscr{D}(\mathfrak{B})$ with independent of $\mathrm{t}, \mathrm{t} \geqslant 0$. Here $\mathfrak{f}:[0, \infty) \times \mathscr{P} \mathscr{C} \rightarrow \mathbb{H}, \mathfrak{g}:[0, \infty) \times \mathscr{P} \mathscr{C} \rightarrow \mathcal{L}_{2}^{0}(\mathbb{K}, \mathbb{H})$ and $\mathfrak{h}:$ $[0, \infty) \times \mathscr{P} \mathscr{C} \times \mathfrak{Z} \rightarrow \mathbb{H}$ are jointly continuous functions. $\Delta x\left(t_{\mathrm{k}}\right)=x\left(\mathrm{t}_{\mathrm{k}}^{+}\right)-x\left(\mathrm{t}_{\mathrm{k}}^{-}\right)$denotes the jump in the state $x$ at time with $\mathrm{I}_{k}(\cdot): \mathbb{H} \rightarrow \mathbb{H}$ determining the size of the jump. $\mathbb{R}_{+}=[0, \infty)$ and $\mathscr{C}(\mathbb{H}, \mathbb{H})$ denote the space of continuous mappings from the topological space $\mathbb{H}$ to the topological space $\mathbb{H}$. Let $\rho(t), \delta(t), \sigma(t) \in \mathscr{C}\left(\mathbb{R}_{+}, \mathbb{R}_{+}\right)$satisfy $t-\rho(t) \rightarrow \infty, t-\delta(t) \rightarrow \infty$ and $t-\sigma(t) \rightarrow \infty$ as $t \rightarrow \infty$, $r(s)=\inf \{s-\rho(s), s-\delta(s), s-\sigma(s), s \geqslant 0\}$ and $r=\inf \{r(s), s \geqslant 0\} . \mathscr{P} \mathscr{C}(J, F)=\{\psi(t): J \rightarrow F \mid \psi(t)$ is a continuous for all but $t_{k} \in \mathbb{R}$ and at these point $t_{k} \in \mathbb{R}, \psi\left(t_{k}^{+}\right)$and $\psi\left(t_{k}^{-}\right)$exist $\left.\psi\left(t_{k}^{+}\right)=\psi\left(t_{k}\right)\right\}$, where $J=[-r, 0], J \subset \mathbb{R}$ is an interval, $F$ is a complete metric space, $\psi\left(t_{k}^{+}\right)$and $\psi\left(t_{k}^{-}\right)$denote the rignt-hand and left-hand limits of the function $\psi(s)$, respectively. The fixed moments of time $t_{k}, k=1,2, \ldots$, satisfy $0<\mathrm{t}_{1}<\cdots<\mathrm{t}_{\mathrm{k}}<\cdots$, and $\lim _{\mathrm{k} \rightarrow \infty} \mathrm{t}_{\mathrm{k}}=\infty$. Espectively, let $\mathscr{P} \mathscr{C}:=\mathscr{P} \mathscr{C}([-\mathrm{r}, 0], \mathbb{H})$ be equipped with the norm

$$
\|\varphi\|_{\mathscr{P} \mathscr{C}}:=\sup _{s \in[-r, 0]}\|\varphi(s)\|_{\mathbb{H}} .
$$

Let $\mathscr{P} \mathscr{C}_{\widetilde{F}_{0}}^{\mathrm{b}}([-\mathrm{r}, 0], \mathbb{H})$ denotes the family of all bounded $\mathfrak{F}_{0}$-measurable, $\mathscr{P} \mathscr{C}$-valued random variables $\phi$, satisfying

$$
\|\phi\|_{\mathcal{L}^{p}}^{p}=\sup _{t \in[-r, 0]} \mathbb{E}\|\phi(t)\|_{\mathbb{H}}^{p}<\infty, p>0
$$

\section{Preliminaries}

In this section, we introduce notations and preliminary results need to establish our results. Let $(\mathbb{H},\|\cdot\|,<\cdot,>)$ and $(\mathbb{K},\|\cdot\|,<\cdot, \cdot>)$ denote two real separable Hilbert spaces, with their vectors norms and their products, respectively. We denote by $\mathcal{L}(\mathbb{K} ; \mathbb{H})$ the set of all linear bounded operators from $\mathbb{K}$ and $\mathbb{H}$, which is equipped with the usual operator norm $\|\cdot\|$. Let $(\Omega, \mathfrak{F}, \mathbb{P})$ be a complete filtered probability space furnished with complete family of right continuous increasing sub $\sigma$-algebras $\left\{\mathfrak{F}_{t}, t \in J\right\}$ satisfying $\mathfrak{F}_{t} \subset \mathfrak{F}$ an $\mathbb{H}$-valued random variable is an $\mathfrak{F}$-measurable function $x(t): \Omega \rightarrow \mathbb{H}$, and a collection of random variable $S=\{x(t, \omega): \Omega \rightarrow \mathbb{H}: t \in J\}$ is called a stochastic process. Let $\beta_{n}(t)(n=1,2, \ldots)$ be a sequence of real valued one-dimensional standard Brownian motions independent of $(\Omega, \mathfrak{F}, \mathbb{P})$. Set $w(t)=\sum_{n=1}^{\infty} \sqrt{\lambda_{n}} \beta_{n}(t) \zeta_{n}(t), t \geqslant 0$, where, $\lambda_{n} \geqslant 0$ are non-negative real numbers and $\left\{\zeta_{n}\right\}(n=1,2, \ldots)$ is complete orthonormal basis in $\mathbb{K}$. Let $\mathrm{Q} \in \mathcal{L}(\mathbb{K}, \mathbb{H})$ be an operator defined by $\mathrm{Q} \zeta_{n}=\lambda_{n} \zeta_{n}$ with finite $\operatorname{Tr}(\mathrm{Q})=\sum_{n=1}^{\infty} \lambda_{n} \leqslant \infty$. Then the above $\mathbb{K}$-valued stochastic process $\mathcal{w}(\mathrm{t})$ is called a $\mathrm{Q}$-Wiener process. Let $\Psi \in \mathcal{L}_{\mathrm{Q}}^{0}(\mathbb{K}, \mathbb{H})$ and define,

$$
\|\Psi\|_{\mathcal{L}_{2}^{0}}^{2}=\operatorname{Tr}\left(\Psi Q \Psi^{*}\right)=\sum_{n=1}^{\infty}\left\|\sqrt{\lambda_{n}} \Psi \zeta_{n}\right\|^{2} .
$$

If $\|\Psi\|_{\mathrm{Q}}<\infty$, then $\Psi$ is known as Q-Hilbert Schmidt operator. For more details on concepts and theory on SDEs, one can refer to the articles $[1,4,9,13,21]$ and references therein.

\subsection{Partial integrodifferential equations in Banach spaces}

In the present section, we recall some definitions, notations and properties of resolvent operator which are needed in the following parts. In what follows, $\mathbb{H}$ will denote a Banach space, $\mathfrak{A}$ and $\mathfrak{B}(t)$ are closed linear operators on $\mathbb{H}$. Y represents the Banach space $\mathscr{D}(\mathfrak{A})$, the domain of operator $\mathfrak{A}$, equipped with the graph norm

$$
\|y\|_{Y}:=\|\mathfrak{A} y\|+\|y\| \text { for } y \in Y \text {. }
$$


The notation $\mathcal{C}([0,+\infty) ; \mathrm{Y})$ stands for the space of all continuous functions from $[0,+\infty)$ into $\mathrm{Y}$. We then consider the following Cauchy problem

$$
\left\{\begin{array}{l}
v^{\prime}(t)=\mathfrak{A} v(t)+\int_{0}^{t} \mathfrak{B}(t-s) v(s) d s \text { for } t \geqslant 0, \\
v(0)=v_{0} \in \mathbb{H}
\end{array}\right.
$$

Definition 3.1 ([11]). A resolvent operator for equation (3.1) is a bounded linear operator valued function $\mathfrak{R}(\mathrm{t}) \in \mathcal{L}(\mathbb{H})$ for $\mathrm{t} \geqslant 0$, satisfying the following properties:

(i) $\mathfrak{R}(0)=\mathrm{I}$ and $\|\mathfrak{R}(\mathrm{t})\| \leqslant M e^{\beta t}$ for some constants $M$ and $\beta$;

(ii) for each $x \in \mathbb{H}, \mathfrak{R}(t) x$ is strongly continuous for $t \geqslant 0$;

(iii) for $x \in Y, \mathfrak{R}(\cdot) x \in \mathcal{C}^{1}([0,+\infty) ; \mathbb{H}) \bigcap \mathcal{C}([0,+\infty) ; Y)$ and

$$
\mathfrak{R}^{\prime}(\mathrm{t}) x=\mathfrak{A} \mathfrak{R}(\mathrm{t}) x+\int_{0}^{t} \mathfrak{B}(t-s) \mathfrak{R}(s) x d s=\mathfrak{R}(t) A x+\int_{0}^{t} \mathfrak{R}(t-s) \mathfrak{B}(s) x d s \text { for } t \geqslant 0 .
$$

For additional detail on resolvent operators, we refer the reader to [11]. In what follows we suppose the following assumptions.

(H1) $\mathfrak{A}$ is the infinitesimal generator of a $C_{0}$-semigroup $\{S(t)\}_{t \geqslant 0}$ on $\mathbb{H}$.

(H2) For all $t \geqslant 0, \mathfrak{B}(t)$ is a continuous linear operator from $\left(Y,\|\cdot\|_{Y}\right)$ into $\left(\mathbb{H},\|\cdot\|_{\mathbb{H}}\right)$. Moreover, there exists an integrable function $c:[0,+\infty) \rightarrow \mathbb{R}^{+}$such that for any $y \in Y, y \rightarrow \mathfrak{B}(\mathrm{t}) \mathrm{y}$ belongs to $\mathrm{W}^{1,1}([0,+\infty) ; \mathbb{H})$ and

$$
\left\|\frac{d}{d t} \mathfrak{B}(t) y\right\|_{\mathbb{H}} \leqslant c(t)\|y\|_{Y} \text { for } y \in Y \text { and } t \geqslant 0 .
$$

Theorem 3.2 ([11]). Assume that hypotheses (H1) and (H2) hold. Then equation (3.1) admits a resolvent operator $(\mathfrak{R}(\mathrm{t}))_{\mathrm{t} \geqslant 0 \text {. }}$

Theorem 3.3 ([11]). Assume that hypotheses (H1) and (H2) hold. Then, the corresponding resolvent operator $\mathfrak{R}(\mathrm{t})$ of equation (3.1) is continuous for $t>0$ in the operator norm, for all $t_{0}>0$, it holds that

$$
\lim _{h \rightarrow 0}\left\|\mathfrak{R}\left(\mathrm{t}_{0}+\mathrm{h}\right)-\mathfrak{R}\left(\mathrm{t}_{0}\right)\right\|=0 .
$$

In the sequel, we recall some results on existence of solutions for the following integrodifferential equation

$$
\left\{\begin{array}{l}
v^{\prime}(t)=\mathfrak{A} v(t)+\int_{0}^{t} \mathfrak{B}(t-s) v(s) d s+q(t) \text { for } t \geqslant 0, \\
v(0)=v_{0} \in \mathbb{H}
\end{array}\right.
$$

where $\mathrm{q}:[0,+\infty) \rightarrow \mathbb{H}$ is a continuous function.

Definition 3.4 ([11]). A continuous function $v:[0,+\infty) \rightarrow \mathbb{H}$ is said to be a strict solution of equation (3.2) if

(i) $v \in \mathfrak{e}^{1}([0,+\infty) ; \mathbb{H}) \cap \mathfrak{e}([0,+\infty) ; Y)$;

(ii) $v$ satisfies equation (3.2) for $t \geqslant 0$.

Remark 3.5. From this definition we deduce that $v(t) \in \mathscr{D}(\mathfrak{A})$, and the function $\mathfrak{B}(t-s) v(s)$ is integrable, for all $t>0$ and $s \in[0,+\infty)$.

Theorem 3.6 ([11]). Assume that (H1)-(H2) hold. If $v$ is a strict solution of equation (3.2), then the following variation of constants formula holds:

$$
v(t)=\mathfrak{R}(\mathrm{t}) v_{0}+\int_{0}^{t} \mathfrak{R}(\mathrm{t}-\mathrm{s}) \mathrm{q}(\mathrm{s}) \mathrm{d} s, \text { for } \mathrm{t} \geqslant 0 .
$$


In this section, we shall get the global attracting and quasi-invariant set and exponential p-th stability of system (2.1), we firstly introduce the concept of the global attracting and quasi-invariant set and exponential p-stability.

Definition 3.7. The set $S \subset \mathbb{H}$ is called a quasi-invariant set of (2.1), if there exist positive constants $k$ and $l$, such that for any initial value $\varphi \in \mathscr{P} \mathscr{C}_{\mathfrak{F}_{0}}^{\mathrm{b}}([-r, 0], \mathbb{H})$, the solution $k x(t, \varphi)+l \in S, t \geqslant 0$.

Definition 3.8. The set $S \subset \mathbb{H}$ is called a global attracting set of (2.1), if for any initial value $\varphi \in$ $\mathscr{P} \mathscr{C}_{\mathfrak{F}_{0}}^{b}([-r, 0], \mathbb{H})$, the solution $k x(t, \varphi)$ converges to $S$ as $t \rightarrow+\infty$. That is, $\operatorname{dist}\left(\chi_{\mathfrak{t}}(0, \varphi), S\right) \rightarrow 0$ as $t \rightarrow \infty$, where $\operatorname{dist}(x, S)=\inf _{y \in S} E\|x-y\|$.

Definition 3.9. The zero solution (or trivial solution) of (2.1) is said to be p-exponentially stable if there exist positive constants $\mu$ and $M>1$, for any initial value $\varphi \in \mathscr{P}_{\mathscr{F}_{0}}^{\mathrm{b}}([-r, 0], \mathbb{H})$, such that

$$
\mathbf{E}\|x(t, \varphi)\|^{p} \leqslant M\|\varphi\|_{\mathcal{L}_{p}} e^{-\mu t}, \quad t \geqslant 0, p \geqslant 2 .
$$

Definition 3.10. The mild solution of (2.1) is said to be almost surely (a.s) exponential stable in $\mathrm{p}^{\text {th }}$ if there exists a positive constant $\mu>0$ such that there is a finite random variable $\varpi e^{-\mu t}$, a.s., $\forall \mathrm{t} \geqslant 0$, or equivalently if

$$
\varlimsup_{\mathrm{tim}} \frac{\log \|x(\mathrm{t})\|^{\mathrm{p}}}{\mathrm{t}} \leqslant-\mu \text { a.s.. }
$$

Lemma 3.11 ([13]). For any a $>0$, assume that there exists some positive constant $\gamma, \gamma_{i},(i=0,1,2, \ldots), \beta_{k}$, $\mathrm{k}=1,2, \ldots$, and a function $\mathfrak{F}(\mathrm{t}) \in \mathscr{P} \mathscr{C}_{\mathfrak{F}_{0}}^{\mathbf{b}}([-\mathrm{r}, 0], \mathbb{H})$ such that

$$
\mathfrak{F}(t) \leqslant\left\{\begin{array}{l}
\gamma_{0} e^{-a t}+\gamma_{1} \int_{0}^{t} e^{-a(t-s)} \sup _{\theta \in[-r, 0]} \mathfrak{F}(s+\theta) d s+\sum_{t_{k}<t} \beta_{k} e^{-a\left(t-t_{k}\right)} \mathfrak{F}\left(t_{k}^{-}\right)+\gamma, t \geqslant 0, \\
\varphi(t), t \in[-r, 0] .
\end{array}\right.
$$

If $\Delta:=\gamma_{1}+\frac{\gamma_{2}}{a}+\sum_{k=1}^{m} \beta_{k}<1$, then there are constant $\lambda \in(0, a)$ and $M>0$ such that

$$
\mathfrak{F}(t) \leqslant M e^{-\lambda t}+(1-\Delta)^{-1} \gamma, t \geqslant 0,
$$

where $\lambda$ and $M$ are determined by

$$
M>\|\varphi\|_{C} \text { and } \mathrm{O}:=\frac{\gamma_{0}}{M}+e^{\lambda t} \frac{\gamma_{2}}{a-\lambda}+\sum_{k=1}^{m} \beta_{k}<1 .
$$

Lemma 3.12 ([13]). For any a $>0$ assume that there exists some positive constant $\gamma, \gamma_{i},(i=0,1,2, \ldots), \beta_{k}$, $\mathrm{k}=1,2, \ldots$, and a function $\mathfrak{F}(\mathrm{t}) \in \mathscr{P} \mathscr{C}_{\mathfrak{F}_{0}}^{\mathrm{b}}([-\mathrm{r}, 0], \mathbb{H})$ such that $\forall \mathrm{t} \geqslant 0$

$$
\mathfrak{F}(t) \leqslant \gamma_{1} \int_{0}^{t} e^{-a(t-s)} \sup _{\theta \in[-r, 0]} \mathfrak{F}(s+\theta) d s+\sum_{t_{k}<t} \beta_{k} e^{-a\left(t-t_{k}\right)} \mathfrak{F}\left(t_{k}^{-}\right)+\gamma, \quad t \geqslant 0 .
$$

If $\Delta:=\frac{\gamma_{2}}{a}+\sum_{k=1}^{m} \beta_{k}<1$, and $\mathfrak{F}(t) \leqslant(1-\Delta)^{-1} \gamma, t \in[-r, 0]$, then $\mathfrak{F}(t) \leqslant(1-\Delta)^{-1} \gamma$.

Now, we give the definition of mild solution for (2.1).

Definition 3.13. A solution process $\{x(t), t \in[0, T], 0 \leqslant T<\infty\}$, is a mild solution of (2.1) if

(1) $x(t)$ is $\mathfrak{F}_{t}$-adapted, $t \geqslant 0$;

(2) $x(t)$ satisfies the integral equation

$$
\begin{aligned}
\chi(t)= & \mathfrak{R}(\mathrm{t}) \chi(0)+\int_{0}^{t} \mathfrak{R}(\mathrm{t}-\mathrm{s}) \mathfrak{f}(s, x(s-\rho(s))) \mathrm{d} s+\int_{0}^{\mathrm{t}} \mathfrak{R}(\mathrm{t}-\mathrm{s}) \mathfrak{g}(s, \chi(s-\delta(s))) \mathrm{d} w(s) \\
& +\int_{0}^{\mathrm{t}} \int_{\mathfrak{Z}} \mathfrak{R}(\mathrm{t}-\mathrm{s}) \mathfrak{h}(s, x(s-\sigma(s)), \eta) \widetilde{\mathfrak{N}}(\mathrm{dt}, \mathrm{d} \eta)+\sum_{0<\mathrm{t}_{\mathrm{k}}<\mathrm{t}} \mathfrak{R}\left(\mathrm{t}-\mathrm{t}_{\mathrm{k}}\right) \mathrm{I}_{\mathrm{k}}\left(\chi\left(\mathrm{t}_{\mathrm{k}}^{-}\right)\right),
\end{aligned}
$$

where $x_{0}(s)=\varphi \in \mathscr{P} \mathscr{C}_{\mathfrak{F}_{0}}^{\mathbf{b}}([-r, 0], \mathbb{H})$. 
In order to obtain our main results, we assume that the following assumptions are satisfied.

(H3) The resolvent operator $(\mathfrak{R}(t))_{t \geqslant 0}$ satisfies the further condition: There exist a constant $M>0$ and real number $\mu>0$ such that

$$
\|\mathfrak{R}(\mathrm{t})\| \leqslant M e^{-\mu t}, \mathrm{t}>0 .
$$

(H4) There exist constant $L_{\mathfrak{f}}>0, L_{\mathfrak{g}}>0$, and $L_{\mathfrak{h}}>0, b_{\mathfrak{f}} \geqslant 0, b_{\mathfrak{g}} \geqslant 0$, and $b_{\mathfrak{h}} \geqslant 0$ such that for any $\mathrm{x}, \mathrm{y} \in \mathscr{P} \mathscr{C}, \mathrm{t} \geqslant 0$,

$$
\begin{aligned}
\|\mathfrak{f}(t, x)-\mathfrak{f}(t, y)\| & \leqslant L_{\mathfrak{f}}\|x-y\|, \quad\|\mathfrak{f}(t, 0)\| \leqslant b_{\mathfrak{f}} \\
\|\mathfrak{g}(t, x)-\mathfrak{g}(t, y)\| & \leqslant L_{\mathfrak{g}}\|x-y\|, \quad\|\mathfrak{g}(t, 0)\| \leqslant b_{\mathfrak{g}} \\
\int_{\mathfrak{Z}}\|\mathfrak{h}(t, x, \eta)-\mathfrak{h}(t, y, \eta)\|^{2} v(d \eta) & \leqslant L_{\mathfrak{h}}^{2}\|x-y\|, \quad\|\mathfrak{h}(t, 0, \eta)\|=0 .
\end{aligned}
$$

(H5) The function $\mathrm{I}_{k} \in \mathscr{C}(\mathbb{H}, \mathbb{H}), k=1,2, \ldots$, satisfies: there exists some positive constants $\mathrm{L}_{\mathrm{I}_{\mathrm{k}}}, \mathrm{b}_{\mathrm{I}_{\mathrm{k}}}$ such that for all $x, y \in \mathbb{H}$,

$$
\left\|I_{k}(x)-I_{k}(y)\right\| \leqslant L_{I_{k}}\|x-y\|,\left\|I_{k}(0)\right\| \leqslant b_{I_{k}} .
$$

(H6) The following inequality

$$
\Delta^{*}=M^{p} \mu^{1-p} L_{\mathfrak{f}}^{p}+C_{p} M^{p} L_{\mathfrak{g}}^{p}\left[\frac{2 \mu(p-1)}{p-2}\right]^{1-\frac{p}{2}}+C_{p} M^{p} L_{\mathfrak{h}}^{p}\left[\frac{2 \mu(p-1)}{p-2}\right]^{1-\frac{p}{2}}+M^{p}\left(\sum_{k=1}^{m} L_{I_{k}}\right)^{\frac{p}{q}}<1
$$

holds for some $\alpha \in(1 / p, 1], p \geqslant 2$ and

$$
\begin{aligned}
& \gamma^{* *}=3^{p-1}\left[M^{p} \mu^{1-p} L_{\mathfrak{f}}^{p}+C_{p} M^{p} L_{\mathfrak{g}}^{p}\left[\frac{2 \mu(p-1)}{p-2}\right]^{1-\frac{p}{2}}+C_{p} M^{p} L_{\mathfrak{h}}^{p}\left[\frac{2 \mu(p-1)}{p-2}\right]^{1-\frac{p}{2}}+M^{p}\left(\sum_{k=1}^{m} L_{I_{k}}\right)^{\frac{p}{q}}\right], \\
& \frac{1}{p}+\frac{1}{q}=1, \quad 0^{0}=1 .
\end{aligned}
$$

\section{Main results}

The first main result of this paper is the following theorem.

Theorem 4.1. Assume that the (H1)-(H6) hold, then

$$
\mathfrak{S}=\left\{\varphi \in \mathscr{P} \mathscr{C}_{\mathfrak{F}_{0}}^{\mathrm{b}}([-r, 0], \mathbb{H}):\|\varphi\|_{\mathcal{L}^{p}}^{p} \leqslant \frac{\gamma^{*}}{1-\Delta^{*}}\right\}
$$

is a global attracting set of the mild solution of (2.1).

Proof. In view of (3.3), taking the expectation, we infer that

$$
\begin{aligned}
\mathbf{E}\|x(\mathrm{t})\|^{\mathrm{p}}= & \mathbf{E} \| \mathfrak{R}(\mathrm{t}) x(0)+\int_{0}^{\mathrm{t}} \mathfrak{R}(\mathrm{t}-\mathrm{s}) \mathfrak{f}(\mathrm{s}, x(\mathrm{~s}-\rho(\mathrm{s}))) \mathrm{d} s+\int_{0}^{\mathrm{t}} \mathfrak{R}(\mathrm{t}-\mathrm{s}) \mathfrak{g}(\mathrm{s}, x(\mathrm{~s}-\delta(\mathrm{s}))) \mathrm{d} w(\mathrm{~s}) \\
& +\int_{0}^{\mathrm{t}} \int_{\mathfrak{Z}} \mathfrak{R}(\mathrm{t}-\mathrm{s}) \mathfrak{h}(\mathrm{s}, x(\mathrm{~s}-\sigma(\mathrm{s})), \eta) \widetilde{\mathfrak{N}}(\mathrm{dt}, \mathrm{d} \eta)+\sum_{0<\mathrm{t}_{\mathrm{k}}<\mathrm{t}} \mathfrak{R}\left(\mathrm{t}-\mathrm{t}_{\mathrm{k}}\right) \mathrm{I}_{\mathrm{k}}\left(x\left(\mathrm{t}_{\mathrm{k}}^{-}\right)\right) \|^{\mathrm{p}} \\
\leqslant & 5^{\mathrm{p}-1} \sum_{5}^{i=1} \mathcal{G}_{\mathrm{i}} .
\end{aligned}
$$


We first evaluate the first term of the right-hand side

$$
\mathcal{G}_{1}=\mathbf{E}\|\mathfrak{R}(\mathrm{t}) \times(0)\|^{p} \leqslant M^{p}\|\varphi\|_{\mathcal{L}^{p}}^{p} e^{-\mu t} .
$$

Using Hölder's inequality and (H4), we obtain that

$$
\begin{aligned}
\mathcal{G}_{2} & =\mathbf{E}\left\|\int_{0}^{t} \mathfrak{R}(\mathrm{t}-\mathrm{s}) \mathfrak{f}(s, x(s-\rho(s))) \mathrm{d} s\right\|^{p} \\
& \leqslant M^{p} \mu^{1-p} L_{\mathfrak{f}}^{p} \int_{0}^{t} e^{-\mu(t-s)} \sup _{-r \leqslant \theta \leqslant 0} \mathbf{E}\|x(s+\theta)\|^{p} d s+M^{p} \mu^{-p} b_{\mathfrak{f}}^{p} .
\end{aligned}
$$

Next, by Hölder's inequality and (H4), and the Burkolder-Davis-Gundy inequalities, we have

$$
\begin{aligned}
\mathcal{G}_{3}= & \mathbf{E}\left\|\int_{0}^{t} \mathfrak{R}(\mathrm{t}-\mathrm{s}) \mathfrak{g}(s, x(s-\delta(s))) \mathrm{d} w(s)\right\|^{p} \\
\leqslant & C_{p} M^{p}\left[\int_{0}^{t}\left(e^{-\mu p(t-s)} \mathbf{E}\|\mathfrak{g}(s, x(s-\delta(s)))\|_{\mathcal{L}_{2}^{0}}^{p}\right)^{\frac{p}{2}} d s\right]^{\frac{p}{2}} \\
\leqslant & C_{p} M^{p}\left[\int_{0}^{t} e^{-\frac{2 \mu(p-1)}{p-2}}(t-s)\right]^{\frac{p}{2}-1} \int_{0}^{t} e^{-\mu(t-s)} \mathbf{E}\|\mathfrak{g}(s, x(s-\delta(s)))\|_{\mathcal{L}_{2}^{0}}^{p} d s \\
\leqslant & C_{p} M^{p} L_{\mathfrak{g}}^{p}\left[\frac{2 \mu(p-1)}{p-2}\right]^{1-\frac{p}{2}} \int_{0}^{t} e^{-\mu(t-s)} \sup _{-r \leqslant \theta \leqslant 0} \mathbf{E}\|x(s+\theta)\|^{p} d s \\
& +C_{p} M^{p} b_{\mathfrak{g}}^{p} \mu^{-1}\left[\frac{2 \mu(p-1)}{p-2}\right]^{1-\frac{p}{2}} .
\end{aligned}
$$

Using Hölder's inequality and (H4), we obtain that

$$
\begin{aligned}
\mathcal{G}_{4} & =\mathbf{E}\left\|\int_{0}^{t} \int_{\mathfrak{Z}} \mathfrak{R}(\mathrm{t}-\mathrm{s}) \mathfrak{h}(s, x(s-\sigma(s)), \eta) \widetilde{\mathfrak{N}}(\mathrm{dt}, \mathrm{d} \eta)\right\|^{p} \\
& \leqslant C_{p} E\left[\int_{0}^{t} \int_{\mathfrak{Z}}\|\mathfrak{R}(\mathrm{t}-\mathrm{s}) \mathfrak{h}(s, x(s-\sigma(s)), \eta)\|^{2} v(\mathrm{~d} \eta) \mathrm{d} s\right]^{\frac{p}{2}} \\
& \leqslant C_{p} M^{p} E\left[\int_{0}^{t} \int_{\mathfrak{Z}} e^{-2 \mu(t-s)}\|\mathfrak{h}(s, x(s-\sigma(s)), \eta)\|^{2} v(d \eta) d s\right]^{\frac{p}{2}} \\
& \leqslant C_{p} M^{p} L_{\mathfrak{h}}^{p}\left[\int_{0}^{t} e^{-\frac{2 \mu(p-1)}{p}}(t-s) e^{-\frac{2 \mu}{p}}(t-s) E\|x(s-\sigma(s))\|^{2} d s\right]^{\frac{p}{2}} \\
& \leqslant C_{p} M^{p} L_{\mathfrak{h}}^{p}\left[\frac{2 \mu(p-1)}{p-2}\right]^{1-\frac{p}{2}} \int_{0}^{t} e^{-\mu(t-s)} \sup _{-r \leqslant \theta \leqslant 0} E\|x(s+\theta)\|^{p} d s .
\end{aligned}
$$

Finally, using Hölder's inequality and (H4), we obtain that

$$
\begin{aligned}
\mathcal{G}_{5} & =\mathbf{E}\left\|\sum_{0<\mathrm{t}_{\mathrm{k}}<\mathrm{t}} \mathfrak{R}\left(\mathrm{t}-\mathrm{t}_{\mathrm{k}}\right) \mathrm{I}_{\mathrm{k}}\left(\mathrm{x}\left(\mathrm{t}_{\mathrm{k}}^{-}\right)\right)\right\|^{\mathrm{p}} \\
& \leqslant \mathbf{E}\left(\sum_{0<\mathrm{t}_{\mathrm{k}}<\mathrm{t}} \mathrm{M}^{\mathrm{p}} \mathrm{e}^{-\mu\left(\mathrm{t}-\mathrm{t}_{\mathrm{k}}\right)}\left[\mathrm{L}_{\mathrm{I}_{\mathrm{k}}}\left\|x\left(\mathrm{t}_{\mathrm{k}}^{-}\right)+\right\| \mathrm{I}_{\mathrm{k}}(0)\|\|\right]\right)^{\mathrm{p}} \\
& \leqslant M^{\mathrm{p}}\left(\sum_{\mathrm{k}=1}^{\mathrm{m}} \mathrm{L}_{\mathrm{I}_{\mathrm{k}}}\right)^{\frac{\mathrm{p}}{q}} \sum_{0<\mathrm{t}_{\mathrm{k}}<\mathrm{t}} \mathrm{L}_{\mathrm{I}_{\mathrm{k}}} e^{-\mathrm{p} \mu\left(\mathrm{t}-\mathrm{t}_{\mathrm{k}}\right)} \mathbf{E}\left\|x\left(\mathrm{t}_{\mathrm{k}}^{-}\right)\right\|^{\mathrm{p}}+\mathrm{M}^{\mathrm{p}}\left(\sum_{\mathrm{k}=1}^{\mathrm{m}} \mathrm{b}_{\mathrm{I}_{\mathrm{k}}}\right)^{\mathrm{p}} .
\end{aligned}
$$


Recalling (4.1), from (4.2)-(4.6), it follows that

$$
\begin{aligned}
\mathbf{E}\|x(t)\|^{p}= & 5^{p-1}\left[M^{p}\|\varphi\|_{L^{p}}^{p} e^{-\mu t}+\left\{M^{p} \mu^{1-p} L_{\mathfrak{f}}^{p}+C_{p} M^{p} L_{\mathfrak{g}}^{p}\left[\frac{2 \mu(p-1)}{p-2}\right]^{1-\frac{p}{2}}\right.\right. \\
& \left.+C_{p} M^{p} L_{\mathfrak{h}}^{p}\left[\frac{2 \mu(p-1)}{p-2}\right]^{1-\frac{p}{2}}\right\} \times \int_{0}^{t} e^{-\mu(t-s)} \sup _{-r \leqslant \theta \leqslant 0} E\|x(s+\theta)\|^{p} d s \\
& \left.+M^{p}\left(\sum_{k=1}^{m} L_{I_{k}}\right)^{\frac{p}{q}} \sum_{0<t_{k}<t} L_{I_{k}} e^{-p \mu\left(t-t_{k}\right)} E\left\|x\left(t_{k}^{-}\right)\right\|^{p}\right]+\gamma^{*} .
\end{aligned}
$$

Let

$$
\begin{aligned}
& \gamma_{0}^{*}=M^{p}\|\varphi\|_{\mathcal{L}^{p}}^{p}, \\
& \gamma_{1}^{*}=M^{p} \mu^{1-p} L_{\mathfrak{f}}^{p}+C_{p} M^{p} L_{\mathfrak{g}}^{p}\left[\frac{2 \mu(p-1)}{p-2}\right]^{1-\frac{p}{2}}+C_{p} M^{p} L_{\mathfrak{h}}^{p}\left[\frac{2 \mu(p-1)}{p-2}\right]^{1-\frac{p}{2}}, \\
& \beta_{k}^{*}=M^{p}\left(\sum_{k=1}^{m} L_{I_{k}}\right)^{\frac{p}{q}} .
\end{aligned}
$$

Obviously, assumption (H6) yields that,

$$
\Delta^{*}=\frac{\gamma_{1}^{*}}{\mu}+\sum_{m}^{k=1} \beta_{\mathrm{k}}^{*}<1,
$$

and since $\varphi \in \mathscr{P} \mathscr{C}_{\mathfrak{F}_{0}}^{\mathrm{b}}([-r, 0], \mathbb{H})$, hence there exist constants $M^{*}>0$ and $\mu^{*} \in(0, \mu)$ such that $M^{*} \geqslant\|\varphi\|_{\mathcal{L}^{p}}^{p}$ and $\mathcal{O}^{*}:=\frac{\gamma_{0}^{*}}{M^{*}}+e^{\mu^{*} r} \frac{\gamma_{1}^{*}}{\mu-\mu^{*}}+\sum_{k=1}^{m} \beta_{k}^{*}<1$. By Lemma 3.11 and inequality (4.7), we infer that

$$
\mathfrak{S}=\left\{\varphi \in \mathscr{P} \mathscr{C}_{\mathfrak{F}_{0}}^{\mathrm{b}}([-r, 0], \mathbb{H}):\|\varphi\|_{\mathcal{L}^{p}}^{p} \leqslant \frac{\gamma^{*}}{1-\Delta^{*}}\right\}
$$

is a global attracting set of the mild solution of (2.1). Hence the proof.

Theorem 4.2. Assume that (H1)-(H6) hold, then

$$
\mathfrak{Q}=\left\{\varphi \in \mathscr{P} \mathscr{C}_{\mathfrak{F}_{0}}^{\mathrm{b}}([-r, 0], \mathbb{H}):\|\varphi\|_{\mathcal{L}^{p}}^{p} \leqslant c_{1}, c_{1}>0\right\}
$$

is a quasi-invariant set of the mild solution of (2.1).

Proof. In view of (3.3), where $\varphi \in \mathfrak{Q}$, we obtain that

$$
\begin{aligned}
E\|x(t)\|^{p}= & 5^{p-1}\left[\left\{M^{p} \mu^{1-p} L_{\mathfrak{f}}^{p}+C_{p} M^{p} L_{\mathfrak{g}}^{p}\left[\frac{2 \mu(p-1)}{p-2}\right]^{1-\frac{p}{2}}\right.\right. \\
& \left.+C_{p} M^{p} L_{\mathfrak{h}}^{p}\left[\frac{2 \mu(p-1)}{p-2}\right]^{1-\frac{p}{2}}\right\} \times \int_{0}^{t} e^{-\mu(t-s)} \sup _{-r \leqslant \theta \leqslant 0} E\|x(s+\theta)\|^{p} d s \\
& \left.+M^{p}\left(\sum_{k=1}^{m} L_{I_{k}}\right)^{\frac{p}{q}} \sum_{0<t_{k}<t} L_{I_{k}} e^{-p \mu\left(t-t_{k}\right)} E\left\|x\left(t_{k}^{-}\right)\right\|^{p}\right]+\gamma^{*}+5^{p-1} M^{p} c_{1} .
\end{aligned}
$$

Let

$$
a^{*}:=5^{p-1} M^{p}, \quad b^{*}=\gamma^{*}
$$

Therefore,

$$
\mathfrak{Q}=\left\{\varphi \in \mathscr{P} \mathscr{C}_{\mathfrak{F}_{0}}^{\mathrm{b}}([-\mathrm{r}, 0], \mathbb{H}):\|\varphi\|_{\mathcal{L}^{p}}^{\mathrm{p}} \leqslant \mathrm{c}_{1}, \mathrm{c}_{1}>0\right\}
$$

is a quasi-invariant set of the mild solution of (2.1). Thus we have completed the proof. 
Corollary 4.3. Assume that assumption (H1)-(H6) with $\mathrm{b}_{\mathfrak{f}}=\mathrm{b}_{\mathfrak{g}}=\mathrm{b}_{\mathrm{I}_{\mathrm{k}}}=0, \mathrm{k}=1,2, \ldots$ hold. Then the mild solution of system (2.1) is exponentially stable in $\mathrm{p}^{\text {th }}$ moment provided that the following inequality

$$
\begin{aligned}
& 1>5^{p-1}\left[M^{p} \mu^{1-p} L_{\mathfrak{f}}^{p}+C_{p} M^{p} L_{\mathfrak{g}}^{p}\left[\frac{2 \mu(p-1)}{p-2}\right]^{1-\frac{p}{2}}+C_{p} M^{p} L_{\mathfrak{h}}^{p}\left[\frac{2 \mu(p-1)}{p-2}\right]^{1-\frac{p}{2}}+M^{p}\left(\sum_{k=1}^{m} L_{I_{k}}\right)^{\frac{p}{q}}\right], \\
& \frac{1}{p}+\frac{1}{q}=1, \quad 0^{0}=1,
\end{aligned}
$$

holds.

Proof. We have

$$
\begin{aligned}
\mathbf{E}\|x(\mathrm{t})\|^{\mathrm{p}}=\mathbf{E} \| & \Re(\mathrm{R}) \chi(0)+\int_{0}^{\mathrm{t}} \mathfrak{R}(\mathrm{t}-\mathrm{s}) \mathfrak{f}(\mathrm{s}, x(s-\rho(s))) \mathrm{d} s+\int_{0}^{\mathrm{t}} \mathfrak{R}(\mathrm{t}-\mathrm{s}) \mathfrak{g}(s, x(s-\delta(s))) \mathrm{d} w(s) \\
& +\int_{0}^{\mathrm{t}} \int_{\mathfrak{Z}} \mathfrak{R}(\mathrm{t}-\mathrm{s}) \mathfrak{h}(\mathrm{s}, x(\mathrm{~s}-\sigma(\mathrm{s})), \eta) \widetilde{\mathfrak{N}}(\mathrm{dt}, \mathrm{d} \eta)+\sum_{0<\mathrm{t}_{\mathrm{k}}<\mathrm{t}} \mathfrak{R}\left(\mathrm{t}-\mathrm{t}_{\mathrm{k}}\right) \mathrm{I}_{\mathrm{k}}\left(x\left(\mathrm{t}_{\mathrm{k}}^{-}\right)\right) \|^{\mathrm{p}} .
\end{aligned}
$$

The right-hand side of the above inequality can be estimated similar to those in the proof of Theorem 4.1 and thus we omit it here. Therefore, we can derive that

$$
\mathbf{E}\|x(\mathrm{t})\|^{p} \leqslant \widetilde{M} e^{-\lambda t}
$$

where $\widetilde{M}>0$ and $\lambda \in(0, \mu)$. That is, the exponential stability in $p^{\text {th }}$ moment for mild solution to system (2.1) is obtained. Hence the proof.

Corollary 4.4. Suppose that all the assumptions of Corollary 4.3 hold with $p>2$, then the mild solution of system (2.1) is almost surely exponentially stable. Moreover, there exists a positive constant $€$ such that

$$
\varlimsup_{t \rightarrow \infty} \frac{\log \|x(t)\|}{t} \leqslant-\frac{\epsilon}{2 p}, \text { a.s.. }
$$

Proof. For each fixed positive real number $\epsilon_{n}, n=1,2, \ldots$,

$$
\begin{aligned}
& \mathbf{P}\left[\sup _{t \in n, n+1}\|x(t)\|^{p}>\epsilon_{n}\right] \leqslant\left(5 / \epsilon_{n}\right)^{p} \mathbf{E}\left[\sup _{t \in n, n+1}\|\mathfrak{R}(t-n)[x(n)]\|^{p}\right] \\
& +\left(5 / \epsilon_{n}\right)^{p} \mathbf{E}\left[\sup _{t \in n, n+1}\|\mathfrak{R}(t-n)[\mathfrak{f}(s, \chi(s-\rho(s))) d s]\|^{p}\right] \\
& +\left(5 / \epsilon_{\mathfrak{n}}\right)^{p} \mathbf{E}\left[\sup _{t \in n, n+1}\|\mathfrak{R}(t-n)[\mathfrak{g}(s, \chi(s-\delta(s))) d w(s)]\|^{p}\right] \\
& +\left(5 / \epsilon_{\mathfrak{n}}\right)^{p} \mathbf{E}\left[\sup _{\mathfrak{t} \in \mathfrak{n}, \mathfrak{n}+1}\|\mathfrak{R}(\mathrm{t}-\mathrm{n})[\mathfrak{h}(s, x(s-\sigma(s)), \eta)] \widetilde{N}(d s, d \eta)\|^{p}\right] \\
& +\left(5 / \epsilon_{n}\right)^{p} \mathbf{E}\left[\sup _{t \in n, n+1}\left\|\sum_{0<t_{k}<t} \mathfrak{R}(t-n) I_{k}\left(x\left(t_{k}^{-}\right)\right)\right\|^{p}\right]=\sum_{5}^{i=1} \Gamma_{i} .
\end{aligned}
$$

In view of Corollary 4.3, we obtain

$$
\begin{aligned}
& \Gamma_{1} \leqslant\left(5 M / \epsilon_{n}\right)^{p} \widetilde{M} e^{-\lambda n}, \quad \quad \Gamma_{2} \leqslant\left(5 M L_{\mathfrak{f}} / \epsilon_{n}\right)^{p} \widetilde{M} e^{\lambda r} e^{-\lambda n}, \quad \Gamma_{3} \leqslant\left(5 M L_{\mathfrak{g}} / \epsilon_{n}\right)^{p} C_{p} \widetilde{M} e^{\lambda r} e^{-\lambda n}, \\
& \Gamma_{4} \leqslant\left(5 M L_{\mathfrak{h}} / \epsilon_{\mathfrak{n}}\right)^{p} \widetilde{M} e^{\lambda r} e^{-\lambda n}, \quad \Gamma_{5} \leqslant\left(\frac{5 M \sum_{k=1}^{m} L_{I_{k}}}{\epsilon_{n}}\right)^{p} \text {. }
\end{aligned}
$$


Thus, there exists a positive real number $\theta$ such that

$$
\mathbf{P}\left[\sup _{t \in \mathfrak{n}, \mathrm{n}+1}\|x(\mathrm{t})\|>\epsilon_{\mathrm{n}}\right] \leqslant\left(\frac{\theta}{\epsilon_{\mathrm{n}}^{\mathrm{p}}}\right) e^{-\frac{\lambda n}{2}} .
$$

Therefore, we have the desired conclusion by a Well-known Borel-Cantelli lemma argument and consequently the proof is complete.

Remark 4.5. If $\mathfrak{h}=0$, the the system (2.1) becomes the following impulsive stochastic differential equations with infinite delays:

$$
\begin{aligned}
\mathrm{d} x(t) & =\left[\mathfrak{A} x(t)+\int_{0}^{t} \mathfrak{B}(t-s) x(s) d s+\mathfrak{f}(t, x(t-\rho(t)))\right] d t+\mathfrak{g}(t, x(t-\delta(t))) d w(t), t \geqslant 0, t \neq t_{k} \\
\Delta x\left(t_{k}\right) & =I_{k}\left(x_{k}^{-}\right), t=t_{k}, k=1,2, \ldots, \\
x_{0}(s) & =\varphi \in \mathscr{P} \mathscr{C}_{\mathfrak{F}_{0}}^{b}([-r, 0], \mathbb{H}), s \in[-r, 0] .
\end{aligned}
$$

Corollary 4.6. Assume that assumptions (H1)-(H6) hold except $\mathbf{( H 4 )}$ with the conditions are imposed on the function $\mathfrak{h}$, then

$$
\mathfrak{S}=\left\{\varphi \in \mathscr{P} \mathscr{C}_{\mathfrak{F}_{0}}^{\mathbf{b}}([-r, 0], \mathbb{H}):\|\varphi\|_{\mathcal{L}^{\mathfrak{p}}}^{\mathrm{p}} \leqslant \frac{\gamma^{* *}}{1-\Delta^{* *}}\right\}
$$

is a global attracting set of the mild solution of (2.1) and

$$
\mathfrak{Q}=\left\{\varphi \in \mathscr{P} \mathscr{C}_{\mathfrak{F}_{0}}^{\mathrm{b}}([-r, 0], \mathbb{H}):\|\varphi\|_{\mathcal{L}^{p}}^{\mathrm{p}} \leqslant \mathrm{c}_{1}, \mathrm{c}_{1}>0\right\}
$$

is a quasi-invariant set of the mild solution of (2.1) if the following inequality

$$
\Delta^{* *}=3^{p-1}\left\{M^{p} \mu^{1-p} L_{\mathfrak{f}}^{p}+C_{p} M^{p} L_{\mathfrak{g}}^{p}\left[\frac{2 \mu(p-1)}{p-2}\right]^{1-\frac{p}{2}}+M^{p}\left(\sum_{k=1}^{m} L_{I_{k}}\right)^{\frac{p}{q}}\right\}<1,
$$

holds for some $\alpha \in(1 / p, 1], p \geqslant 2$ and

$$
\gamma^{* *}=3^{p-1}\left[M^{p} \mu^{1-p} L_{\mathfrak{f}}^{p}+C_{p} M^{p} L_{\mathfrak{g}}^{p}\left[\frac{2 \mu(p-1)}{p-2}\right]^{1-\frac{p}{2}}+M^{p}\left(\sum_{k=1}^{m} L_{I_{k}}\right)^{\frac{p}{q}}\right], \quad \frac{1}{p}+\frac{1}{q}=1, \quad 0^{0}=1 .
$$

Proof. The proof of this corollary is similar to that of Theorems 4.1 and 4.2, and one can easily prove the solution of system (4.10) and hence, it is omitted.

\section{Example}

We consider the following impulsive stochastic partial integrodifferential equation with Poisson jumps:

$$
\begin{aligned}
\mathrm{d} x(t)= & {\left[\frac{\partial^{2}}{\partial z^{2}} x(t, \zeta)+\int_{0}^{t} \mathfrak{B}(t-s)\left[x(t, \zeta)+u_{1} x(t-r, \zeta)\right]\right] d t+u_{2} x(t-r, \zeta) d w(t) } \\
& +\int_{\mathfrak{Z}} u_{3} x(t-r, \eta, \zeta) \widetilde{N}(d t, d \eta), 0 \leqslant z \leqslant \pi, t \geqslant 0, t \neq t_{k}, \\
\Delta x\left(t_{k}\right)= & I_{k}\left(x\left(t_{k}^{-}\right)\right)=\frac{u_{4}}{k^{2}} x\left(t_{k}^{-}\right), t=t_{k}, \\
x(t, 0)= & x(t, \pi)=0, t \geqslant 0, \\
x_{0}(t, \zeta)= & \varphi(t) \in \mathscr{P} \mathscr{C}_{\mathfrak{F}_{0}}^{b}\left([-r, 0], \mathcal{L}^{2}([0, \pi])\right),-r \leqslant t \leqslant 0,
\end{aligned}
$$


where $u_{i}>0, i=1,2,3,4$, are constants, $w(t)$ denotes the standard cylindrical Wiener process. Let $\mathbb{H}=\mathbb{K}=\mathcal{L}^{2}([0, \pi])$ and $e_{\mathfrak{n}}=\sqrt{\frac{2}{\pi}} \sin (n x), n=1,2,3, \ldots$. Then $\left(e_{n}\right)_{\mathfrak{n} \in \mathbb{N}}$ is a complete orthonormal basis in $\mathbb{K}$. Define $\mathfrak{A}: \mathscr{D}(\mathfrak{A}) \subset \mathbb{H} \rightarrow \mathbb{H}$ by $\mathfrak{A}=\frac{\partial^{2}}{\partial z^{2}}$, with domain $\mathscr{D}(\mathfrak{A})=\mathbb{H}^{2}([0, \pi]) \cap \mathbb{H}_{0}^{1}([0, \pi])$ and

$$
\mathfrak{A} z=-\sum_{n=1}^{\infty} n^{2}<z, e_{n}>e_{n}, \mathscr{D}(\mathfrak{A}) .
$$

$\mathfrak{A}$ is the infinitesimal generator of a strongly continuous semigroup $\{\mathrm{S}(\mathrm{t})\}_{\mathrm{t} \geqslant 0}$ on $\mathbb{H}$, which is given by

$$
S(t) \phi=-\sum_{\infty}^{n=1} e^{-n^{2} t}<\phi, e_{n}>e_{n}, \phi \in \mathscr{D}(\mathfrak{A}) .
$$

Let $\mathfrak{B}: \mathscr{D}(\mathfrak{B}) \subset \mathbb{H} \rightarrow \mathbb{H}$ be the operator defined by $\mathfrak{B}(t): \mathfrak{B}(t) z$ for $t \geqslant 0$ and $z \in \mathscr{D}(\mathfrak{A})$. Define operators $\mathfrak{f}(\mathrm{t}, \phi)(\zeta)=\mathrm{u}_{1} \phi(\mathrm{t}-\mathrm{r}, \zeta), \zeta \in[0, \pi], \mathrm{t} \geqslant 0, \mathfrak{g}(\mathrm{t}, \phi)(\zeta)=\mathrm{u}_{2} \phi(\mathrm{t}-\mathrm{r}, \zeta), \zeta \in[0, \pi], \mathrm{t} \geqslant 0 \mathfrak{h}(\mathrm{t}, \phi)(\zeta)=$ $\mathrm{u}_{3} \phi(t-r, \zeta), \zeta \in[0, \pi], \mathrm{t} \geqslant 0, \mathrm{I}_{\mathrm{k}}\left(x\left(\mathrm{t}_{\mathrm{k}}^{-}\right)\right)(\zeta)=\frac{\mathrm{u}_{4}}{\mathrm{k}^{2}} x\left(\mathrm{t}_{\mathrm{k}}^{-}\right)$. Then assumptions (H3)-(H6) are satisfied with $\mathrm{L}_{\mathfrak{f}}=\vartheta_{1}, \mathrm{~L}_{\mathfrak{g}}=\vartheta_{2}, \mathrm{~L}_{\mathfrak{h}}=\vartheta_{3}, \mathrm{~L}_{\mathrm{I}_{\mathfrak{k}}}=\vartheta_{4}$. Let $\mathrm{p}=2$, then we have

$$
\gamma_{1}^{*}=M^{p} \mu^{1-p} L_{\mathfrak{f}}^{p}+C_{p} M^{p} L_{\mathfrak{g}}^{p}\left[\frac{2 \mu(p-1)}{p-2}\right]^{1-\frac{p}{2}}+C_{p} M^{p} L_{\mathfrak{h}}^{p}\left[\frac{2 \mu(p-1)}{p-2}\right]^{1-\frac{p}{2}}+M^{p}\left(\sum_{k=1}^{m} L_{I_{k}}\right)^{\frac{p}{q}}:=\Theta .
$$

From Theorem 4.1, we conclude that $\mathfrak{S}=\left\{\varphi \in \mathscr{P} \mathscr{C}_{\widetilde{\mathfrak{F}}_{0}}^{\mathrm{b}}([-r, 0], \mathbb{H}):\|\varphi\|_{\mathcal{L}^{p}}^{p} \leqslant \frac{\gamma^{*}}{1-\Delta^{*}}\right\}$ is the global attracting set of the system (5.1) provided that $\Theta<1$. In particular, when $\vartheta_{1}=\vartheta_{2}=\vartheta_{3}=0$ and $\Theta<1$, by Theorem 4.2 , the mild solution of system (5.1) is exponential stable in mean square.

\section{Acknowledgment}

I would like to thank the referees and the editor for their careful reading and their valuable comments.

\section{References}

[1] A. Anguraj, K. Ravikumar, Existence and stability of impulsive stochastic partial neutral functional differential equations with infinite delays and Poisson jumps, Discontinuity, Nonlinearity, and Complexity, 9 (2020), 245-255. 1, 3

[2] A. Anguraj, K. Ramkumar, E. M. Elsayed, Existence, uniqueness and stability of impulsive stochastic partial neutral functional differential equations with infinite delays driven by a fractional Brownian motion, Discontinuity, Nonlinearity, and Complexity, 9 (2020), 327-337. 1

[3] D. Applebaum, Lévy processes and stochastic calculus, Cambridge University Press, Cambridge, (2009). 1

[4] G. Arthi, J. H. Park, H. Y. Jang, Exponential stability for second order neutral stochastic differential equations with impulses, Internat. J. Control, 88 (2015), 1300-1309. 3

[5] T. Caraballo, K. Liu, Exponential stability of mild solutions of stochastic partial differential equations with delays, Stochastic Anal. Appl., 17 (1999), 743-763. 1

[6] D. Chalishajar, K. Ravikumar, A. Anguraj, Impulsive-integral inequalities for attracting and quasi-invariant sets of neutral stochastic partial functional integrodifferential equations with impulsive effects, J. Nonlinear Sci. Appl., 13 (2020), 284-292. 1

[7] H. Chen, Impulsive-integral inequality and exponential stability for stochastic partial differential equations with delays, Statist. Probab. Lett., 80 (2010), 50-56. 1

[8] H. Chen, The existence and exponential stability stability for neutral stochastic partial differential equations with infinite delay and Poisson jump, Indian J. Pure Appl. Math., 46 (2015), 197-217.

[9] G. Da Prato, J. Zabczyk, Stochastic Equations in Infinite Dimensions, Cambridge University Press, Cambridge, (1992). 1, 3

[10] P. Duan, Y. Ren, Attracting and quasi-invariant sets of neutral stochastic integrodifferential equations with impulses driven by fBm, Adv. Differ. Equ., 2017 (2017), 1-15. 1

[11] R. C. Grimmer, Resolvent operators for integral equations in a Banach space, Trans. Amer. Math. Soc., 273 (1982), 333-349. 3.1, 3.1, 3.2, 3.3, 3.4, 3.6 
[12] D. D. Huan, R. P. Agarwal, Global attracting and quasi-invariant sets for stochastic Volterra-Levin equations with jumps, Dyn. Contin. Discrete Impuls. Syst., Ser. A, Math. Anal., 21 (2014), 343-353. 1

[13] D. D. Huan, R. P. Agarwal, Asymptotic behavior, attracting and quasi-invariant sets for impulsive neutral SPFDE driven by Lévy noise, Stoch. Dyn., 18 (2018), 21 pages. 1, 3, 3.11, 3.12

[14] C. Knoche, Mild solutions of SPDEs driven by Poisson noise in infinite dimensions and their dependence on initial conditions, Thesis dissertation, Bielefeld University, (2005). 1

[15] V. Laksmikantham, D. D. Baĭnov, P. S. Simeonov, Theory of Impulsive Differential Equations, World Scientific, Singapore, (1989). 1

[16] B. Li, The attracting set for impulsive stochastic difference equations with continuous time, Appl. Math. Lett., 25 (2012), 1166-1171. 1

[17] Z. Li, Global attracting and quasi-invariant sets of impulses neutral stochastic functional differential equations driven by fBm, Neurocomputing, 117 (2016), 620-627. 1

[18] D. Li, D. Xu, Existence and global attractivity of periodic solution for impulsive stochastic Volterra-Levin equations, Electron. J. Qual. Theory Differ. Equ., 2012 (2012), 1-12. 1

[19] K. Liu, A. Truman, A note on almost sure exponential stability for stochastic partial functional differential equations, Statist. Probab. Lett., 50 (2000), 273-278. 1

[20] S. Long, L. Teng, D. Xu, Global attracting set and stability of stochastic neutral partial functional differential equations with impulses, Statist. Probab. Lett., 82 (2012), 1699-1709. 1

[21] B. Øksendal, Stochastic Differential Equations, Springer, Berlin, Heidelberg, (2003). 1, 3

[22] K. Ramkumar, A. Anguraj, Impulsive-integral inequalities for attracting and quasi-invariant sets of neutral stochastic integrodifferential equations with impulsive effects, J. Appl. Nonlinear Dyn., 9 (2020), 513-523. 1

[23] L. Wang, D. Li, Impulsive-integral inequalities for attracting and quasi-invariant sets of impulsive stochastic partial differential equations with infinite delays, J. Inequal. Appl., 2013 (2013), 11 pages. 1 